\title{
Síndrome da hipoventilação central congênita: estudo de caso
}

\author{
Congenital central hypoventilation syndrome: case study
}

Síndrome de hipoventilación central congénita: estudio de caso

Patrícia Marina Galego¹, Débora Renata Galego ${ }^{2 *}$, lasmin Barufaldi Prette ${ }^{2}$, Regina Célia Ajej Pires de Albuquerque ${ }^{2}$, Andressa Regina Galego², Patricia de Barros Viegas Anno².

\section{RESUMO}

Objetivo: Descrever caso de Síndrome da Hipoventilação Central Congênita (SHCC) em lactente. Detalhamento de caso: Lactente de 1 mês e 20 dias admitido em unidade de emergência com relato de cianose perioral e de extremidades desencadeada por choro e tosse presentes desde o nascimento e piora progressiva nos 7 dias antecedentes à admissão no serviço. Descartadas etiologias infecciosa, pulmonar, cardíaca, malformações de sistema nervoso central, erros inatos do metabolismo, distúrbios hidroeletrolíticos e neuromusculares. Apesar de sedação contínua e ventilação mecânica otimizada, apresentou episódios frequentes de queda da saturação de oxigênio (StO2) de e bradicardia, principalmente quando manipulado. As gasometrias arteriais de controle indicaram aumento importante da pressão parcial de gás carbônico, atingindo até 130 , com padrão mantido, mesmo com StO2 normal e de $\mathrm{FIO} 2$ próxima a $100 \%$. A hipótese diagnóstica de hipoventilação central congênita foi confirmada por meio da identificação GENE PHOX2B em HETEROZIGOSE (1 cópia), variante patogênica da doença. Considerações finais: O diagnóstico precoce e as melhorias na ventilação domiciliar, acompanhados do aperfeiçoamento dos métodos de monitoramento cardiorrespiratório e de ventilação artificial, têm permitido aos pacientes atingir a idade adulta com qualidade de vida satisfatória. Os avanços tecnológicos da engenharia biomédica prometem aos pacientes maior independência e autonomia.

Palavras-chave: Hipoventilação central congênita, Síndrome de Ondine, Gene PHOX2B, Expansão repetida de polialaninas (PARM), Expansão de repetição não-polialanina (NPARM).

\begin{abstract}
Objective: To describe a case of Central Congenital Hypoventilation Syndrome (CCHS) in an infant. Case details: Infant aged 1 month and 20 days admitted to the emergency unit with a report of perioral and extremity cyanosis triggered by crying and coughing present since birth and progressive worsening in the 7 days prior to admission to the service. Discarded infectious, pulmonary, cardiac etiologies, central nervous system malformations, inborn errors of metabolism, hydroelectrolytic and neuromuscular disorders. Despite continuous sedation and optimized mechanical ventilation, he had frequent episodes of decreased oxygen saturation (StO2) and bradycardia, especially when handled. Control arterial blood gases indicated a significant increase in the partial pressure of carbon dioxide, reaching up to 130 , with a maintained pattern, even with normal StO2 and $\mathrm{FIO} 2$ close to $100 \%$. The diagnostic hypothesis of congenital central hypoventilation was confirmed by identifying GENE PHOX2B in HETEROZYGOSIS (1 copy), a pathogenic variant of the disease. Final considerations: Early diagnosis and improvements in home ventilation, together with the improvement of methods of cardiorespiratory monitoring and artificial ventilation, have allowed patients to reach adulthood with a satisfactory quality of life. Technological advances in biomedical engineering promise patients greater independence and autonomy.
\end{abstract}

Keywords: Congenital central hypoventilation, Ondine syndrome, PHOX2B gene, Polyalanine repeat expansion (PARM), Non-polyalanine repeat expansion (NPARM).

\section{RESUMEN}

Objetivo: Describir un caso de Síndrome de Hipoventilación Congénita Central (SHCC) en un lactante. Detalles del caso: Lactante de 1 mes y 20 días ingresado en urgencias con reporte de cianosis perioral y de

${ }^{1}$ Centro Universitário Barão de Mauá (CUBM), Ribeirão Preto - SP. *E-mail: debora.galegoo@hotmail.com

2 Faculdade de Medicina de São José do Rio Preto (FAMERP), São José do Rio Preto - SP. 
extremidades desencadenada por llanto y tos presente desde el nacimiento y empeoramiento progresivo en los 7 días previos al ingreso al servicio. Se descartan etiologías infecciosas, pulmonares, cardíacas, malformaciones del sistema nervioso central, errores innatos del metabolismo, alteraciones hidroelectrolíticas y neuromusculares. A pesar de la sedación continua y la ventilación mecánica optimizada, presentaba frecuentes episodios de disminución de la saturación de oxígeno (StO2) y bradicardia, especialmente al manipularlo. La gasometría arterial control indicó un aumento significativo de la presión parcial de dióxido de carbono, llegando hasta 130, con un patrón mantenido, incluso con StO2 normal y FiO2 cercano al 100\%. La hipótesis diagnóstica de hipoventilación central congénita se confirmó al identificar GENE PHOX2B en HETEROZIGOSIS (1 copia), una variante patogénica de la enfermedad. Consideraciones finales: El diagnóstico precoz y las mejoras en la ventilación domiciliaria, junto con la mejora de los métodos de monitorización cardiorrespiratoria y ventilación artificial, han permitido que los pacientes lleguen a la edad adulta con una calidad de vida satisfactoria. Los avances tecnológicos en ingeniería biomédica prometen a los pacientes una mayor independencia y autonomía.

Palabras clave: Hipoventilación central congénita, Síndrome de Ondine, Gen PHOX2B, Expansión repetida de polialanina (PARM), Expansión repetida sin polialanina (NPARM).

\section{INTRODUÇÃO}

A Síndrome da Hipoventilação Central Congênita (SHCC), também conhecida como Síndrome de Ondine, é uma doença rara, de etiologia genética, secundária a mutações no gene da homeobox pareada (PHOX2B). Nestes pacientes, o controle autonômico da respiração é falho, principalmente durante o sono NREM, de forma que não apresentam as respostas fisiológicas normais à hipóxia ou à hipercapnia, como sintomas de dispnéia, desconforto ou ansiedade, e sinais de taquipneia, queimação nasal ou uso de músculos respiratórios acessórios (MALONEY MA, et al., 2018).

A síndrome da hipoventilação central congênita foi primariamente descrita em 1970 e o gene PHOX2B, definidor da doença, foi descoberto apenas em 2003, impulsionando, a partir de então, possibilidade de testes genéticos, diagnóstico de certeza da doença e a busca por tratamentos (BERRY-KRAVIS EM, et al., 2006).

Até 2010, mais de 1000 casos geneticamente confirmados de síndrome da hipoventilação central congênita foram documentados em todo o mundo, com uma prevalência estimada de 1 em 200.000 nascidos vivos na França e de 1 em 148.000 nascidos vivos no Japão (SASAKI A, et al., 2019).

De acordo com Berry-Kravis EM, et al. (2006), a principal característica da SHCC é hipoventilação alveolar, mas a disfunção autonômica se manifesta também em outros sistemas orgânicos, como gastrointestinais, cardiovascular e endócrino. O objetivo deste estudo foi descrever um caso de síndrome da hipoventilação central congênita.

\section{DETALHAMENTO DO CASO}

T.G.C., 1 mês e 20 dias, admitido na unidade de emergência de um hospital universitário no noroeste paulista com relato de cianose perioral e de extremidades, desencadeada por choro e tosse desde 0 nascimento, com piora 1 semana antes da admissão no serviço. Apresentou, em atendimento inicial, queda da saturação e versão ocular, seguidos por movimentos clônicos de membros e apneia, com interrupção da crise clínica após a administração intravenosa de diazepam e de intubação orotraqueal.

Foram descartadas causas infecciosas, cardíacas, pulmonares, distúrbios hidroeletrolíticos, doenças neuromusculares, malformações de sistema nervoso central e erros inatos do metabolismo. $O$ eletroencefalograma de 1 hora evidenciou distúrbio epileptiforme em regiões anteriores. A polissonografia foi inconclusiva devido condições técnicas e clínicas que não permitiram realização completa do exame.

O paciente permaneceu internado na Unidade de Terapia Intensiva, em ventilação mecânica e sedação contínua, monitorizado, com episódios frequentes de queda da saturação de oxigênio (StO2) e bradicardia, principalmente quando manipulado, apesar da ventilação mecânica otimizada e curarização.

As gasometrias arteriais de controle indicaram aumento importante da pressão parcial de gás carbônico, atingindo até 130, com padrão mantido, mesmo com StO2 normal e de $\mathrm{FIO} 2$ próxima a 100\%. 
Durante a internação, apresentou várias paradas cardiorrespiratórias, revertidas após manobras de ressuscitação. Evoluiu com melhora clínica, permanecendo com traqueostomia e mantendo saturação de oxigênio adequada em ar ambiente. Feita a hipótese de hipoventilação central congênita, confirmada por reação em cadeia da polimerase (PCR) seguido por sequenciamento tipo Sanger, com identificação da variante patogênica: GENE PHOX2B em heterozigose (1 cópia).

O menor atualmente reside em domicílio, em regime de home care, e tem consultas periódicas para acompanhamento neuropediátrico, genético, cardiovascular e pediátrico.

\section{DISCUSSÃO}

A mutação do gene PHOX2B provoca uma falha no controle autonômico da respiração e também outras disautonomias. A mutação do gene PHOX2B é definidor síndrome da hipoventilação central congênita (MALONEY MA, et al., 2018).

Na Síndrome da Hipoventilação Central Congênita, o drive respiratório central é comprometido, causando hipoventilação, insuficiência respiratória, hipercapnia e hipoxemia. Essa disfunção é mais grave durante o sono, principalmente durante o sono não-REM, quando o acionamento ventilatório é controlado quase inteiramente pela entrada metabólica e pelo controle automático da respiração. No sono REM, a hipoventilação é menos intensa e variável durante a vigília (MALONEY MA, et al., 2018; BISHARA J, et al., 2018).

Os quimiorreceptores centrais estão intactos e funcionantes, visto que os indivíduos com SHCC são capazes de aumentar a ventilação minuto e o volume corrente em resposta ao exercício. O movimento passivo dos membros inferiores nos estados de sono e vigília aumenta a ventilação alveolar, confirmando que a via pela qual os mecanorreceptores estimulam a respiração é ativa e funcionante (MALONEY MA, et al., 2018); BISHARA J, et al., 2018).

O gene PHOX2B é composto por uma sequência de repetição de 20 alaninas no éxon 3 (genótipo 20/20). Nos pacientes com síndrome da hipoventilação central congênita existem mutações de expansão repetida de polialaninas (PARMs) que passam a conter entre 24 a 33 repetições de alaninas, formando os genótipos mais comuns da doença, que são: 20/25, 20/26 e 20/27 PARMs. Mais de 90\% dos casos de síndrome da hipoventilação central congênita ocorrem por uma dessas PARM no gene PHOX2B. Os demais pacientes (10\%) possuem uma mutação de expansão de repetição não-polialanina (NPARM) nos éxons 1, 2 ou 3 devido a mutações missense, nonsense ou frameshift no gene PHOX2B. Deleções completas e parciais do gene PHOX2B foram descritas em indivíduos com características clínicas sugestivas de SHCC e podem representar outras formas de mutações causadoras de doença (MALONEY MA, et al., 2018; BISHARA J, et al., 2018; BERRY-KRAVIS EM, et al., 2006; SASAKI A, et al., 2019 ; YE G, et al., 2019; MAGALHÃES J., et al., 2015; TRANG H., et al., 2014; WILLIAMS P, et al., 2014.

A síndrome da hipoventilação central congênita é tipicamente herdada em um padrão autossômico dominante, embora a maioria das mutações ocorra de novo (novas mutações germinativas decorrentes dos gametas dos pais). O mosaicismo somático e germinativo tem sido relatado em pais assintomáticos de crianças com SHCC, assim, a prevalência de mutações de novo pode ser superestimada. Penetrância incompleta e expressividade variável foram observadas em pacientes com PARMs 20/24 e 20/25 e algumas mutações NPARM. Esta variabilidade obriga os provedores a manter um alto índice de suspeita de mutações PHOX2B em membros assintomáticos da família e ressalta a importância dos testes e de aconselhamento genético para as famílias (BERRY-KRAVIS EM, et al., 2006; SASAKI A, et al., 2019).

Todos os pacientes com suspeita de Síndrome da Hipoventilação Central Congênita devem ser submetidos a exames de análise genética da mutação do gene PHOX2B para confirmação diagnóstica. $\mathrm{O}$ tipo de mutação do gene não só confirma o diagnóstico como também prediz o fenótipo da doença, a gravidade de comprometimento respiratório e distúrbios autonômicos associados (MALONEY MA, et al., 2018; BISHARA J, et al., 2018).

Pacientes com PARMs mais curtos (20/24 e 20/25) raramente necessitam de suporte ventilatório contínuo e podem se apresentar como SHCC de "início tardio". Indivíduos com PARMs mais longos (20/27 a 20/33) e 
indivíduos com NPARMs frequentemente requerem suporte ventilatório de 24 horas e também estão associados à doença de Hirschsprung e à tumores de células da crista neural (SASAKI A, et al., 2019; BYERS HM, et al., 2018; MATERA I, et al., 2004; PATTYN A., et al., 1999; DUBREUIL V., et al., 2008; UNGER SA, et al., 2017).

Estabelecido o diagnóstico, os pacientes com Síndrome da Hipoventilação Central Congênita devem ser rastreados para disfunção autonômica associada. É necessário descartar doenças neuromusculares ou metabólicas subjacentes. Outras causas de hipoventilação devem ser excluídas, de preferência comqu métodos não invasivos de monitoramento de oxigenação e ventilação, como a polissonografia. Outros exames complementares relevantes são: radiografia de tórax, eletrocardiograma e ecocardiograma, a fim de avaliar anormalidades de área cardíaca, ritmo cardíaco, hipertensão pulmonar e cor pulmonale. Em alguns casos, considerar tomografia computadorizada de tórax para avaliar doença pulmonar primária, ressonância magnética e/ou tomografia computadorizada do cérebro e de tronco cerebral para rastrear lesões anatômicas. A avaliação laboratorial de erros inatos do metabolismo deve ser realizada em pacientes que não foram submetidos à triagem neonatal abrangente (BISHARA J, et al., 2018; BYERS HM, et al., 2018; ZAIDI S, et al., 2018; NAGASHIMADA M, et al., 2012).

De acordo com o Quadro 1, podemos observar que os sinais de desregulação autonômica, a depender do fenótipo, se estendem além do controle desordenado da respiração e podem ter manifestações em diversos sistemas, como cardiovascular, gastrointestinal, oftalmológico, alterações neurocognitivas, tumores de crista neural, desregulação de temperatura diaforese anormal, dentre outras. Portanto, a vigilância periódica desses pacientes é imprescindível para a identificação precoce de alterações nestes sistemas (NAGASHIMADA M, et al., 2012; AMIEL J, et al., 2008; LOMBARDO RC, et al., 2017).

Quadro 1- Manifestações orgânicas de disautonomia associadas à SHCC.

\begin{tabular}{|c|c|}
\hline Sistemas orgânicos & Manifestações clínicas \\
\hline Cardiovascular & $\begin{array}{c}\text { Diminuição da variabilidade da frequência cardíaca; } \\
\text { Diminuição da resposta da frequência cardíaca ao exercício; } \\
\text { Arritmias potencialmente fatais; } \\
\text { Anormalidades da pressão arterial; } \\
\text { Síncope; } \\
\text { Bradicardia sinusal, pausas sinusais, assistolia transitória; } \\
\text { Prolongamento do intervalo QT; }\end{array}$ \\
\hline Gastrointestinal & $\begin{array}{l}\text { Doença de Hirschsprung (20\%); } \\
\text { Constipação intestinal severa; } \\
\text { Dismotilidade esofágica; }\end{array}$ \\
\hline Tumores da crista neural & $\begin{array}{c}\text { Neuroblastomas; } \\
\text { Ganglioneuromas; } \\
\text { Ganglioneuroblastomas; }\end{array}$ \\
\hline Oftalmológico & $\begin{array}{l}\text { Anisocoria; } \\
\text { Resposta pupilar anormal; } \\
\text { Estrabismo; }\end{array}$ \\
\hline Neurocognitivo & $\begin{array}{c}\text { Crises epilépticas; } \\
\text { Déficit cognitivo; } \\
\text { Distúrbios de aprendizagem; } \\
\text { Alteração de percepção da dor; }\end{array}$ \\
\hline Endocrinológico & $\begin{array}{l}\text { Hipoglicemia; } \\
\text { Hiperglicemia; } \\
\text { Hiperinsulinemia; }\end{array}$ \\
\hline Outros & $\begin{array}{c}\text { Desregulação da temperatura; } \\
\text { Diaforese anormal; } \\
\text { Alterações faciais; } \\
\text { Inflexão inferior da borda lateral do lábio superior; }\end{array}$ \\
\hline
\end{tabular}

Fonte: Galego PM, et al., 2021; dados extraídos de Maloney MA, et al., 2018; Nagashimada M, et al., 2012; Amiel J, et al., 2008; Lombardo RC, et al., 2017. 
O objetivo no manejo do paciente com a Síndrome da Hipoventilação Central Congênita é garantir ventilação e oxigenação adequadas em todos os momentos ao longo da vida por meio do uso de ventilação mecânica durante o sono ou por suporte contínuo 24 horas, se necessário (BISHARA J, et al., 2018; CHEN ML, et al., 2005).

Devido ao rápido crescimento cerebral e à vulnerabilidade à hipóxia presente nos primeiros anos de vida, a maioria dos lactentes e das crianças jovens devem ser mecanicamente ventiladas por meio de traqueostomia. Os neonatos devem inicialmente ser ventilados 24 horas por dia, devido ciclos de sono-vigília curtos e imprevisíveis. A necessidade de ventilação deve ser reavaliada periodicamente, uma vez que alguns passam a depender da ventilação apenas em períodos do dia (MALONEY MA, et al., 2018; BISHARA J, et al., 2018; CHEN ML, et al., 2005; NAGASHIMADA M, et al., 2012). Apenas oxigênio suplementar é insuficiente, pois essa terapia melhorará a oxigenação sem abordar a hipoventilação, que é a origem do problema (DUBREUIL V, et al., 2008).

É necessária muita atenção durante os períodos de doença aguda, pois os pacientes com SHCC não apresentam taquipnéia, desconforto respiratório ou dispneia em resposta ao aumento da carga respiratória, hipercapnia ou hipóxia, impostos pela infecção respiratória. Durante esses períodos, os pacientes podem necessitar de suporte ventilatório em tempo integral e/ou ajustes do ventilador. Em caso de deterioração aguda ou crônica da condição clínica, a primeira hipótese diagnóstica deve ser por hipoventilação. Este é um ponto crítico de educação para pacientes, famílias e médicos (MALONEY MA, et al., 2018; CHEN ML, et al., 2005; WANG A, et al., 2018).

Os pulmões dos pacientes com Síndrome da Hipoventilação Central Congênita são geralmente saudáveis, permitindo uma variedade de opções ventilatórias, como: ventilação com pressão positiva via traqueostomia, ventilação não invasiva com pressão positiva ou estimulação do diafragma (ZAIDI S, et al., 2018; CHEN ML, et al., 2005).

A ventilação com pressão positiva via traqueostomia é o modo recomendado para neonatos e crianças pequenas, pois garante oxigenação e ventilação adequadas em todos os momentos, promovendo o melhor desenvolvimento neurológico para esses pacientes. As crianças mais velhas e com fenótipos mais leves que necessitam de ventilação assistida apenas durante o sono são candidatas ideais para o modo ventilação não invasiva com pressão e deve ser usado em modo cronometrado para garantir a ventilação adequada. Em neonatos e crianças pequenas, o uso de ventilação com máscara pode causar hipoplasia da face devido à imaturidade dos ossos faciais e da cartilagem, não devendo ser usada durante os períodos de vigília, e aumenta o risco de lesão da pele (ZAIDI S, et al., 2018; CHEN ML, et al., 2005; WANG A, et al., 2018).

A entrega de ar seco pode provocar a irritação da mucosa nasal, congestão nasal crônica e epistaxe recorrente, amenizados com uso de um umidificador no circuito. A estimulação do diafragma é um modo de ventilação mecânica que usa o próprio diafragma do paciente como ventilador. Os pacientes que necessitam de suporte ventilatório durante o dia podem usá-lo, proporcionando liberdade do ventilador e mobilidade. 0 uso de estimulação do diafragma pode permitir a decanulação da traqueostomia nos pacientes dependentes de ventilador somente no período do sono (ZAIDI S, et al.; 2018; CHEN ML, et al., 2005); WANG A, et al., 2018).

Os candidatos à estimulação de diafragma devem ter pouca ou nenhuma doença pulmonar intrínseca, nervos frênicos intactos, função normal do diafragma e não devem ser obesos, visto que a obesidade pode interferir na transmissão do sinal. Um transmissor externo gera um sinal de frequência de rádio que é enviado através de uma antena externa para receptores subcutâneos bilaterais. Esses receptores convertem o sinal em corrente elétrica que é transportada por fios de aço inoxidável até eletrodos nos nervos frênicos, estimulando-os e resultando na contração do diafragma e, assim, na ventilação pulmonar (CHEN ML, et al., 2005; WANG A, et al., 2018).

O funcionamento da estimulação do diafragma necessita de uma abordagem conservadora e gradual, permitindo que a criança permaneça relaxada enquanto se acostuma à nova sensação. As configurações são vagarosamente aumentadas até que seja obtida a contração adequada do diafragma. A oximetria de pulso contínua e a PETCO2 são usadas para monitorar a oxigenação e a ventilação, enquanto a criança é observada no hospital por aproximadamente 48 horas (MALONEY MA, et al., 2018). 
Inicialmente, a estimulação é limitada entre 60 a 90 minutos por dia, por fadiga diafragmática e diminuição da resposta ao estímulo. A duração da estimulação é aumentada semanalmente em cerca de 30 minutos por dia até ser atingida uma estimulação contínua de 4 horas. Nesse momento, o tempo de estimulação é aumentado em 1 hora por semana, até atingir funcionamento máximo de 14 a 16 horas por dia. Em certos casos, o estimulador diafragmático pode não ser adequado (durante as doenças respiratórias agudas) ou/nem viável (durante ou após uma grande cirurgia), quando outros modos de ventilação podem ser necessários. Existe a possibilidade de decanulação da traqueostomia em alguns pacientes com Síndrome da Hipoventilação Central Congênita com um fenótipo mais leve e que necessitem apenas de suporte ventilatório mecânico durante o sono (ZAIDI S, et al., 2018).

A identificação de mutações NPARMs no gene PHOX2B e maior entendimento da Síndrome da Hipoventilação Central Congênita ajudará a elucidar os mecanismos genéticos pelos quais as mutações no gene PHOX2B podem resultar em doença. A literatura ainda é restrita e no contexto científico brasileiro, desafiadora. $O$ diagnóstico precoce $e$ as melhorias da ventilação domiciliar, acompanhados do aperfeiçoamento dos métodos de monitoramento cardiorrespiratório e de ventilação artificial, têm permitido aos pacientes atingirem a idade adulta com qualidade de vida satisfatória. Os avanços da engenharia biomédica prometem aos pacientes mobilidade e independência ainda maiores.

\section{REFERÊNCIAS}

1. AMIEL J, et al. Hirschsprung disease, associated syndromes and genetics: A review. J. Med. Genet. 2008 ; 45: 1-14.

2. BISHARA J, et al. The genetics of congenital central hypoventilation syndrome: clinical implications. The Aplication of Clinical Genetics, 2018; 11: 135-144.

3. BERRY-KRAVIS EM, et al. Congenital Central Hypoventilation Syndrome. PHOX2B Mutations and Phenotype. American Journal of Respiratory and Critical Care Medicine, 2006; 174: 1139-44.

4. BYERS HM, et al. Expanding the phenotype of congenital central hypoventilation syndrome impacts management decisions. Am J Med Genet Part A. 2018;176A: 1398-1404.

5. CHEN ML, et al. Diaphragm pacers as a treatment for congenital central hypoventilation syndrome. Expert Ver. Med. Devices, 2005; 2: 577-85.

6. DUBREUIL V, et al. A human mutation in Phox2b causes lack of $\mathrm{CO} 2$ chemosensitivity, fatal central apnea, and specific loss of parafacial neurons. National Academy of Sciences of the United States of America, 2008 105(3): 1067-1072.

7. LOMBARDO RC, et al. Variable phenotype in a novel mutation in PHOX2B. American Journal of Medical Genetics Part A, 2017; 173(6): 1705-1709.

8. MAGALHÃES J., et al. Late-onset congenital central hypoventilation syndrome and a rare PHOX2B gene mutation. Sleep and Breathing, 2015; 19(1): 55-60.

9. MALONEY MA, et al. Congenital central hypoventilation syndrome: diagnosis and management. Expert Review of Respiratory Medicine, 2018, 12: 283-292.

10. MATERA I, et al. PHOX2B mutations and polyalanine expansions correlate with the severity of the respiratory phenotype and associated symptoms in both congenital and late onset central hypoventilation syndrome. Journal of Medical Genetics, 2004; 41(5): 373-380.

11. NAGASHIMADA $M$, et al. Autonomic neurocristopathy-associated mutations in PHOX2B dysregulate Sox10 expression. J. Clin. Invest. 2012; 122: 3145-58.

12. PARRA A, et al. Early Diagnosis Non-Invasive Management in a Newborn with Classic Congenital Central Hypoventilation Syndrome. Am. J. Respir. Crit. Care Med, 2019;199: A4978.

13. PATTYN A., et al. The homeobox gene Phox2b is essential for the development of autonomic neural crest derivatives. Nature, 1999; 399(6734): 366-370.

14. SASAKI A, et al. Novel PHOX2B mutations in congenital central hypoventilation syndrome. Pediatrics International, 2019; 61: 393-396.

15. TRANG H., et al. European Central Hypoventilation Syndrome Consortium. Proceedings of the fourth international conference on central hypoventilation. Orphanet Journal of Rare Diseases, 2014; 9: 194.

16. UNGER SA, et al. A case of "abnormally abnormal" hypoxic ventilatory responses: A novel NPARM PHOX2B gene mutation. Journal of Clinical Sleep Medicine, 2017; 13(08): 1013-1015.

17. WANG A, et al. Obstructive Sleep Apnea in Patients With Congenital Central Hypoventilation Syndrome Ventilated by Diaphragm Pacing Without Tracheostomy. J Clin Sleep Med. 2018;14(2): 261-264.

18. WILLIAMS P, et al. Outcomes in multifocal neuroblastoma as part of the neurocristopathy syndrome. Pediatrics, 2014; 134(2): E611-E616.

19. YE G, et al. A Novel c.676_677insG PHOX2B Mutation in Congenital Central Hypoventilation Syndrome. Journal of Clinical Medicine, 2019; 15: 509-13.

20. ZAIDI S, et al. Congenital central hypoventilation syndrome: An overview of etiopathogenesis, associates pathologies, clinical presentation, and management. Autonomic Neuroscience: Basic \& Clinical, 2018; 210: 1-9. 